\title{
Similar short-term clinical response to high- dose versus low-dose methotrexate in monotherapy and combination therapy in patients with rheumatoid arthritis
}

Sytske Anne Bergstra ${ }^{1 *}$ D, Cornelia F. Allaart ${ }^{1}$, Rosaline van den Berg ${ }^{2}$, Arvind Chopra ${ }^{3}$, Nimmisha Govind ${ }^{4}$, Tom W. J. Huizinga' ${ }^{1}$ and Robert B. M. Landewe $e^{5,6}$

\begin{abstract}
Background: Aiming at rapid decrease of disease activity, there has been a trend to start with higher doses of methotrexate (MTX) in patients newly diagnosed with rheumatoid arthritis (RA), both as monotherapy and in combination with other antirheumatic drugs. We aimed to study the relationship between clinical response and MTX dose as monotherapy or combination therapy in patients with early RA.

Methods: Disease-modifying anti-rheumatic drug (DMARD)-naive patients with early RA, from a large international observational database, the METEOR database, were selected if MTX was part of their initial treatment. Patients were divided into four groups: MTX monotherapy, MTX + convention synthetic (cs)DMARDs, MTX + glucocorticoids or MTX + biologic (b)DMARDs. MTX dose was dichotomized: low dose $\leq 10 \mathrm{mg} /$ week; high dose $\geq 15 \mathrm{mg} / \mathrm{week}$. Linear mixed model analyses for the Disease Activity Score (DAS), DAS in 28 joints (DAS28) and Health Assessment Questionnaire ( $\mathrm{HAQ}$ ) were performed in each medication group, with MTX dose and time as covariates. Outcomes were assessed from baseline until 3-6 months follow up. Associations were adjusted for potential confounding by indication using propensity score (PS) modelling.
\end{abstract}

Results: For patients starting MTX monotherapy $(n=523)$, MTX + csDMARDs $(n=266)$ or MTX + glucocorticoids ( $n=615$ ), the PS-adjusted effects of MTX dose (high versus low) on the DAS, DAS28 and HAQ were small and not clinically meaningful. Patients starting MTX + bDMARDs were disregarded due to low numbers $(n=11)$.

Conclusions: In patients newly diagnosed with RA, no clinical benefit of high compared to low initial MTX doses was found for MTX monotherapy or for MTX combination therapy with csDMARDs or glucocorticoids.

Keywords: Rheumatoid arthritis, Methotrexate, Dose, Outcome measures, Combination therapy

\section{Background}

Methotrexate (MTX) is the anchor drug in the treatment of rheumatoid arthritis (RA). Current recommendations for MTX monotherapy suggest initiation of $15 \mathrm{mg} /$ week orally, and escalation with $5 \mathrm{mg} / \mathrm{month}$ to $25-30 \mathrm{mg} /$ week or the highest tolerable dose $[1,2]$. There are no specific recommendations for use of MTX in combination with other antirheumatic drugs (glucocorticoids, conventional

\footnotetext{
*Correspondence: s.a.bergstra@lumc.nl

${ }^{1}$ Department of Rheumatology, Leiden University Medical Centre, Leiden,

The Netherlands

Full list of author information is available at the end of the article
}

synthetic disease-modifying antirheumatic drugs (csDMARDs) and/or biologic DMARDs (bDMARDs)). Many studies have shown faster reduction of disease activity, quicker improvement in physical functioning and less radiographic evidence of damage progression on MTX combination therapy than on MTX monotherapy [3-6]. It is questionable whether a higher initial MTX dose in combination with other effective medication is more effective in the short term than a lower initial MTX dose. The CONCERTO study compared four treatment arms with different MTX doses $(2.5,5,10$ or $20 \mathrm{mg} /$ week) in combination with adalimumab $40 \mathrm{mg} / 2$ weeks in patients with 
early RA [7]. More patients achieved the Disease Activity Score in 28 joints (DAS28) low disease activity or remission status with increasing MTX doses over 26 weeks. However, radiographic progression and the Health Assessment Questionnaire (HAQ) scores were similar in the various arms. The proportions of patients achieving low disease activity or remission were similar in the MTX $10 \mathrm{mg} /$ week and MTX $20 \mathrm{mg} /$ week arms.

Recently, a meta-regression analysis of trials in patients with recent onset RA showed that higher initial MTX doses were not associated with better short-term clinical outcomes, neither with MTX monotherapy, nor with MTX in combination with bDMARDs or glucocorticoids [8].

In the current study we aimed to assess the influence of MTX dose on disease outcomes and physical functioning in an international cohort with real-life data. We hypothesized that in patients newly diagnosed with RA the initial MTX dose as monotherapy or in combination with other csDMARDs, bDMARDs or glucocorticoids will not determine short-term outcomes.

\section{Methods \\ Data selection}

Data from the international, observational, Measurement of Efficacy of Treatment in the Era of Outcome in Rheumatology (METEOR) database were used, which has been described previously [9]. For the current study, we selected all DMARD-naive patients with early RA and symptom duration $<5$ years, with $\geq 1$ follow-up visits after 3-6 months. At both baseline and follow-up visits, patients had to have at least one of the following outcome measures: DAS, DAS28, erythrocyte sedimentation rate (ESR), C-reactive protein (CRP) or HAQ. MTX had to be part of the initial treatment as monotherapy or in combination with other csDMARDs/bDMARDs/glucocorticoids. Variation in dose was allowed (e.g. step-up MTX dose or step-down prednisone dose) but no change in medication type was allowed between initial treatment and the followup visit after 3-6 months. Since the METEOR database consists of observational data gathered in clinical practice, there are irregular time intervals between follow-up visits and the number of follow-up visits differs per patient. Therefore, the last visit within 3-6 months after treatment initiation, meeting all inclusion and exclusion criteria was defined for each patient, and all follow-up visits between baseline and this last follow-up visit were selected. In order to take into account step-up dosing schedules, the MTX dose prescribed at the final visit before 3 months follow up was used.

\section{Statistical analysis}

Patients were analysed in four groups, based on initial MTX strategy: (1) MTX monotherapy, (2) MTX + other csDMARDs, (3) MTX + glucocorticoid (+/- additional
csDMARDs) or (4) MTX + bDMARD (+/- additional csDMARDs). Missing data were imputed using multivariate normal multiple imputation (30 cycles). Linear mixed model (LMM) analyses were performed to assess the effectiveness of MTX dose on the outcome measures DAS, DAS28 and HAQ, within the four groups. To account for irregular time intervals, random intercept and slope were added to each model, with "independence" covariance matrix. MTX dose was dichotomized (low dose $\leq 10 \mathrm{mg} /$ week; high dose $\geq 15 \mathrm{mg} /$ week). Time in days between baseline and each follow-up visit was added as continuous variable.

Differences in environmental and patient characteristics may affect the initial MTX dose, and therefore may have caused confounding by indication. To adjust for potential confounding, the propensity score (PS) was calculated in the imputed dataset, using multiple probit regression analysis based on observed baseline patient and environmental characteristics [10]. Several PS models were tested and compared for the best data fit in all 30 imputations. Representing the probability of receiving an intervention given the observed baseline variables, the PS was then added as covariate adjustment to the LMM analyses. Details on the PS are given in Additional file 1. All LMM analyses were performed with and without PS, to see whether confounding by indication was present. All analyses were performed using STATA SE 14 (StataCorp LP).

\section{Results}

From the METEOR database, 1438 patients (3193 visits) were selected: 523 patients (1120 visits) started MTX monotherapy, 266 patients (581 visits) started MTX + csDMARDs, 615 patients (1416 visits) started MTX + glucocorticoids and 11 patients (26 visits) started MTX + bDMARDs (Fig. 1). Detailed information on concomitant treatment is presented in Additional file 2. Patients originated from 20 different countries, with $94 \%$ of data originating from India, South-Africa, Portugal, the Netherlands, the USA, Ireland and Mexico. Too few patients started MTX + bDMARDs to perform meaningful analyses. In addition, 23 patients (50 visits) who started MTX $12.5 \mathrm{mg}$ /week (the intermediate dose) were disregarded. Baseline characteristics of the other patients are shown in Table 1. There was a trend over time to start higher MTX doses (Additional file 3).

Because physicians were free to choose their own disease activity measure, the DAS and DAS28 based on ESR were missing in $40 \%$ and $35 \%$ of all visits, respectively. However, an official disease activity measure was only unavailable in $4 \%$ of all visits and a disease activity measure component was only unavailable in $0.3 \%$ of all visits. 


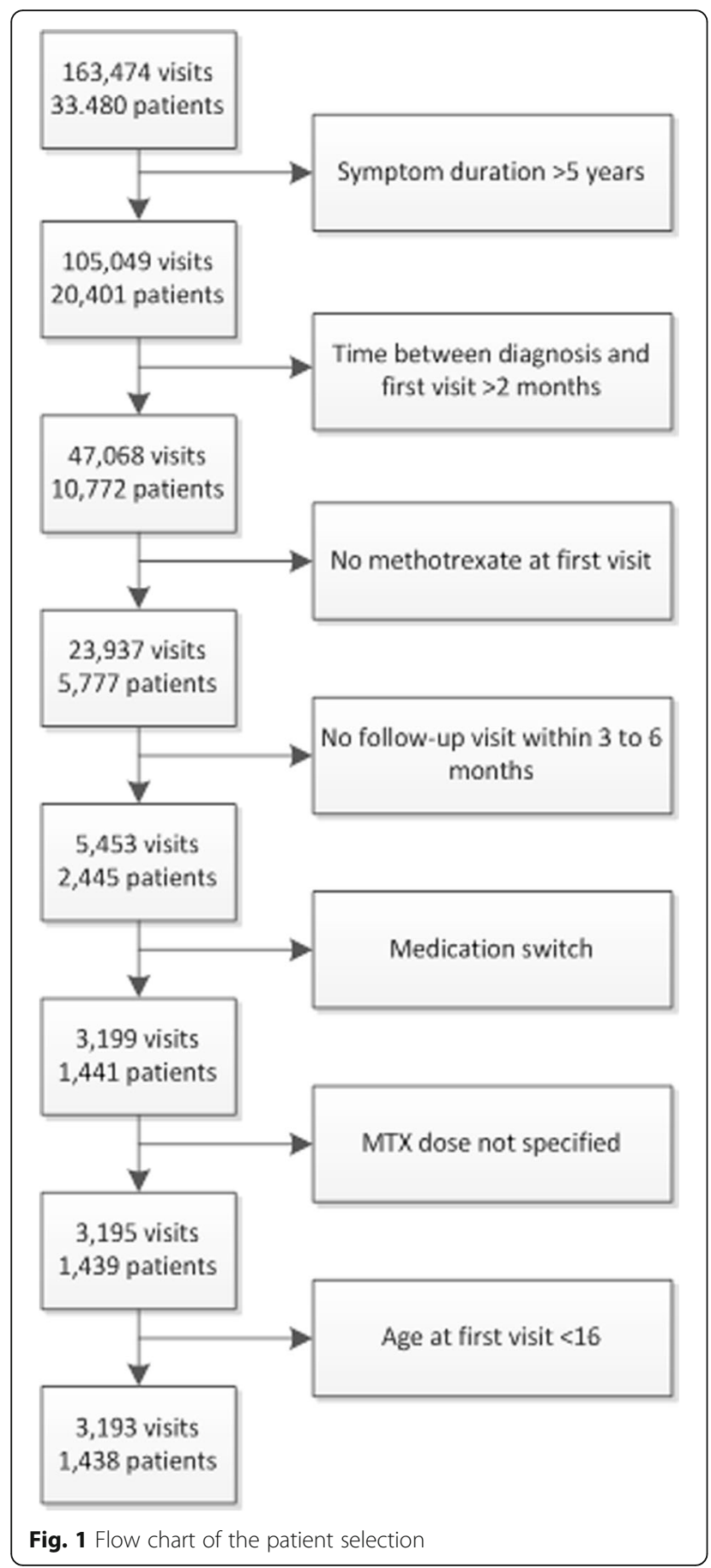

In Table 2, the PS-adjusted and unadjusted coefficients for the association between initial MTX dose and outcomes within 3-6 months follow-up are presented, stratified by treatment group. For patients starting MTX monotherapy, MTX + csDMARDs or MTX + glucocorticoids, the PS-adjusted effects of MTX dose (high vs low) on DAS, DAS28 and HAQ were small and not clinically meaningful. For example, in the MTX monotherapy group, $\beta(95 \% \mathrm{CI})$ for the outcome DAS was 0.070
$(-0.15$; 0.29), indicating an increase in DAS of 0.070 for a high versus a low MTX dose.

The unadjusted main associations between MTX dose and outcomes were often in an opposite direction and/ or much larger than the PS-adjusted associations, suggesting that confounding by indication indeed plays a role and that it has been (at least partly) corrected for by adjusting for the PS. Two sensitivity analyses were performed: one excluding the country, which added most patients to the analyses (India) and one excluding all patients with a symptom duration $>2$ years; both resulted in similar outcomes (data not shown).

\section{Discussion}

In this study based on daily practice treatment decisions in patients newly diagnosed with RA, we did not identify clinical benefit of high compared to low MTX starting doses in monotherapy or in combination with csDMARDs or glucocorticoids: high initial MTX doses did not result in greater improvement in the DAS, DAS28 or HAQ compared to low initial MTX doses. Co-medication with csDMARDs or glucocorticoids did not influence this effect. In an earlier meta-regression analysis we showed that also in clinical trials there was no early clinical benefit of a high compared to a low MTX starting dose [8].

We observed a trend over time in daily practice to start higher MTX doses. In particular patients receiving comedication with glucocorticoids as initial treatment were prescribed higher MTX doses, possibly as the rheumatologist estimated their RA to be more severe. Although we used PS to adjust for baseline differences that may have influenced the treatment decisions of the rheumatologist as well as the outcomes, intangible or unmeasured baseline differences may still affect the results.

We assessed response to treatment within 3-6 months, since current recommendations advise a treat-to-target strategy in which medication is intensified or changed as soon as possible if treatment is not effective. The more rapid onset of action of glucocorticoids as co-treatment may mask any effect of the initial dose of slow-acting MTX $[3,11]$. As demonstrated in clinical trials, this appears also to be true for initial treatment with bDMARDs and MTX, but as this is a rare initial treatment in daily practice, we were unable to investigate this further. However, also with MTX monotherapy a higher dose was not more effective than a low dose. The most likely explanation is in the pharmacokinetics of MTX, where stable availability of active MTX polyglutamates seems independent of the weekly MTX dose [12].

This study has potential limitations. The effect of MTX dose was assessed within three subgroups depending on the use and type of co-medication, but within each group, variations in type, number and dose of additional drugs in individual patients could 
Table 1 Baseline characteristics per treatment group, non-imputed data

\begin{tabular}{|c|c|c|c|c|c|c|}
\hline \multirow[b]{2}{*}{ Age at first visit (years) } & \multicolumn{2}{|c|}{$\begin{array}{l}\text { MTX monotherapy }(n=523) \\
\text { Number }\end{array}$} & \multicolumn{2}{|c|}{$\begin{array}{l}\text { MTX + csDMARDs }(n=266) \\
\text { Number }\end{array}$} & \multicolumn{2}{|c|}{$\begin{array}{l}\text { MTX + glucocorticoids }(n=615) \\
\text { Number }\end{array}$} \\
\hline & 522 & $47.9(13.1)$ & 264 & $44.6(10.9)$ & 479 & $48.3(14.8)$ \\
\hline Gender (\% female) & 520 & 78 & 266 & 83 & 609 & 81 \\
\hline Body mass index & 281 & $26.6(6.7)$ & 184 & $27.6(6.3)$ & 272 & $27.2(6.0)$ \\
\hline Symptom duration at diagnosis median (IQR) & 451 & $365(169-731)$ & 266 & $730(365-1095)$ & 482 & $458(181-1095)$ \\
\hline Rheumatoid factor (\% positive) & 511 & 77 & 263 & 84 & 585 & 81 \\
\hline ACPA (\% positive) & 300 & 72 & 98 & 85 & 342 & 76 \\
\hline Erosions present (\% positive) & 305 & 40 & 62 & 55 & 293 & 52 \\
\hline ESR & 462 & $56.5(33.0)$ & 241 & $69.3(31.7)$ & 543 & $59.5(35.5)$ \\
\hline CRP & 415 & $33.1(33.9)$ & 219 & $40.3(35.5)$ & 515 & $37.7(37.1)$ \\
\hline $\mathrm{HAQ}$ & 439 & $1.0(0.6)$ & 249 & $1.1(0.6)$ & 506 & $1.3(0.7)$ \\
\hline DAS & 314 & $3.7(1.2)$ & 189 & $4.0(0.96)$ & 347 & $3.9(1.2)$ \\
\hline DAS 28 & 340 & $5.7(1.5)$ & 192 & $6.2(1.2)$ & 415 & $6.0(1.5)$ \\
\hline MTX dose (\% high dose) & 523 & 28 & 266 & 14 & 615 & 46 \\
\hline Follow-up duration (days) & 523 & $134(28)$ & 266 & $135(28)$ & 615 & $139(31)$ \\
\hline
\end{tabular}

Data per number of patients are means (SD), unless indicated otherwise. MTX methotrexate, csDMARDs conventional synthetic disease-modifying antirheumatic drugs, IQR inter quartile range, $A C P A$ anti-citrullinated protein antibodies, ESR erythrocyte sedimentation rate, CRP C-reactive protein, HAQ Health Assessment Questionnaire, DAS Disease Activity Score

influence efficacy. However, previous clinical trials have shown comparable disease outcomes of various combination therapies and dosing schedules for many drugs are fixed $[13,14]$. We dichotomized MTX dosage, and defined MTX $>15 \mathrm{mg} /$ week as high dose, which is used in current recommendations, but is still an arbitrary cutoff. The results might have been slightly different with other cutoffs. In addition, MTX was mostly administered orally, and uptake can vary between individuals. We have no further data on the number and timing of patients who might have switched to subcutaneous treatment. The results might have been different with subcutaneous administration of MTX. Moreover, although we are unaware of any evidence that the response to methotrexate could differ between the countries included in the analysis, we took into account the potential influence of country on our outcomes and adjusted for potential country differences by adding country to the propensity score.

Since real-world data were used, no formal procedures were taken to control the quality of clinical assessments, which may have led to more noise compared to clinical trial data. However, our data are in line with previous findings [8].

Table 2 Unadjusted and propensity score-adjusted results of the linear mixed model analyses to investigate the effectiveness of high versus low methotrexate doses on disease activity (DAS and DAS28) and physical functioning (HAQ), stratified per medication group

\begin{tabular}{|c|c|c|c|}
\hline & $\begin{array}{l}\text { DAS } \\
\beta(95 \% \mathrm{Cl}) \\
\end{array}$ & $\begin{array}{l}\text { DAS28 } \\
\beta(95 \% \text { Cl) }\end{array}$ & $\begin{array}{l}\text { HAQ } \\
\beta(95 \% \text { Cl) }\end{array}$ \\
\hline \multicolumn{4}{|c|}{ Methotrexate monotherapy (number of patients $=522$, number of visits $=1090$ ) } \\
\hline MTX dose group PS-adjusted & $0.070(-0.15 ; 0.29)$ & $0.12(-0.19 ; 0.43)$ & $0.060(-0.09 ; 0.21$ \\
\hline MTX dose group unadjusted & $-0.63(-0.79 ;-0.47)$ & $-0.90(-0.13 ;-0.67)$ & $0.16(0.055 ; 0.26)$ \\
\hline \multicolumn{4}{|c|}{ Methotrexate + csDMARDs (number of patients $=262$, number of visits $=567$ ) } \\
\hline MTX dose group PS-adjusted & $0.051(-0.23 ; 0.33)$ & $0.024(-0.37 ; 0.42)$ & $-0.0058(-0.20 ; 0.19)$ \\
\hline MTX dose group unadjusted & $-0.18(-0.44 ; 0.072)$ & $-0.28(-0.63 ; 0.072)$ & $0.092(-0.085 ; 0.27)$ \\
\hline \multicolumn{4}{|c|}{ Methotrexate + oral glucocorticoid (+/-csDMARDs) (number of patients $=615$, number of visits $=1403$ ) } \\
\hline MTX dose group PS-adjusted & $-0.047(-0.26 ; 0.16)$ & $-0.16(-0.44 ; 0.12)$ & $-0.028(-0.16 ; 0.11)$ \\
\hline MTX dose group unadjusted & $-0.42(-0.56 ; 0.28)$ & $-0.74(-0.93 ;-0.55)$ & $0.13(0.045 ; 0.22)$ \\
\hline
\end{tabular}

Time is modelled in days between the baseline visit and each follow-up visit. Low dose is the reference category. MTX dose group is a binary variable with low dose $\leq 10 \mathrm{mg} /$ week and high dose $\geq 15 \mathrm{mg} /$ week. DAS Disease Activity Score, DAS 28 Disease Activity Score in 28 joints, HAQ Health Assessment Questionnaire, PS propensity score, 95\% Cl 95\% confidence interval, MTX methotrexate, csDMARDs conventional synthetic disease-modifying antirheumatic drug 


\section{Conclusion}

In conclusion, these real-world data show that in patients newly diagnosed with RA, a higher MTX dose with or without other CsDMARD or glucocorticoids does not result in better clinical efficacy after 3-6 months compared to a low dose. This seems to contradict a general trend over time to start with higher MTX doses. Without apparent early benefit, higher initial MTX dosages may introduce more side effects, which may jeopardise drug retention. However, since side effects were not measured in the METEOR database, we could not assess this. On the other hand, starting a low MTX dose may induce delays in suppression of disease activity and in the introduction of additional therapies, as up to $23 \%$ of patients have been found to require higher dosage and up to $56 \%$ have not been found to achieve low disease activity with MTX [15]. For the moment, our results suggest that although with MTX monotherapy there may be other considerations, rheumatologists should consider a low instead of a high initial MTX dose, in particular when prescribed in combination with other csDMARDs or glucocorticoids, and further modify treatment according to a treat-totarget protocol.

\section{Additional files}

\section{Additional file 1: Extended description of the propensity score} estimation. (DOCX $20 \mathrm{~kb}$ )

Additional file 2: Detailed information on concomitant treatment. (DOCX $14 \mathrm{~kb}$ )

Additional file 3: Frequency of methotrexate doses over time in newly diagnosed patients in the METEOR database. (DOCX $15 \mathrm{~kb}$ )

\section{Abbreviations \\ bDMARD: Biologic disease-modifying anti-rheumatic drug; CRP: C-reactive protein; csDMARD: Conventional synthetic disease-modifying anti-rheumatic drug; DAS(28): Disease Activity Score in 28 joints; ESR: Erythrocyte sedimentation rate; HAQ: Health assessment questionnaire; LMM: Linear mixed model; METEOR: Measurement of Efficacy of Treatment in the Era of Outcome in Rheumatology; MTX: Methotrexate; PS: Propensity score; RA: Rheumatoid arthritis}

\section{Acknowledgements}

Not applicable.

\section{Funding}

No funding was received for this project.

\section{Availability of data and materials}

The dataset used and/or analysed during the current study are available from the corresponding author on reasonable request.

\section{Authors' contributions}

$S A B, C A, R v d B$ and $R L$ made substantial contributions to conception and design of the study, data analysis and interpretation of data. SAB, CA and $R L$ drafted the manuscript. $C A, A C, N G$ and $T H$ made substantial contributions to data acquisition. All authors revised, read and approved the final manuscript.

\section{Ethics approval and consent to participate}

Since this study used only anonymized, observational data, no ethics approval or informed consent was needed.

\section{Consent for publication}

Not applicable.

\section{Competing interests}

The authors declare that they have no competing interests.

\section{Publisher's Note}

Springer Nature remains neutral with regard to jurisdictional claims in published maps and institutional affiliations.

\section{Author details}

${ }^{1}$ Department of Rheumatology, Leiden University Medical Centre, Leiden, The Netherlands. ${ }^{2}$ Department of Rheumatology, Erasmus University Medical Center, Rotterdam, The Netherlands. ${ }^{3}$ Center for Rheumatic Diseases, Pune, India. ${ }^{4}$ University of the Witwatersrand, Johannesburg, South Africa.

${ }^{5}$ Amsterdam Rheumatology \& Immunology Center, Amsterdam, The Netherlands. ${ }^{6}$ Zuyderland Medical Center, Heerlen, The Netherlands.

Received: 26 June 2017 Accepted: 7 November 2017

Published online: 22 November 2017

\section{References}

1. Smolen JS, Landewe R, Breedveld FC, Buch M, Burmester G, Dougados M, et al. Eular recommendations for the management of rheumatoid arthritis with synthetic and biological disease-modifying antirheumatic drugs: 2013 update. Ann Rheum Dis. 2014;73:492-509.

2. Visser K, Katchamart W, Loza E, Martinez-Lopez JA, Salliot C, Trudeau J, et al. Multinational evidence-based recommendations for the use of methotrexate in rheumatic disorders with a focus on rheumatoid arthritis: integrating systematic literature research and expert opinion of a broad international panel of rheumatologists in the $3 e$ initiative. Ann Geophys. 2009:68:1086-93.

3. Goekoop-Ruiterman YP, de Vries-Bouwstra JK, Allaart CF, van Zeben D, Kerstens PJ, Hazes JM et al. Clinical and radiographic outcomes of four different treatment strategies in patients with early rheumatoid arthritis (the BeSt study): a randomized, controlled trial. Arthritis Rheum. 2005;52:3381-90.

4. Breedveld FC, Weisman MH, Kavanaugh AF, Cohen SB, Pavelka K, van Vollenhoven $\mathrm{R}$ et al. The Premier Study: a multicenter, randomized, doubleblind clinical trial of combination therapy with adalimumab plus methotrexate versus methotrexate alone or adalimumab alone in patients with early, aggressive rheumatoid arthritis who had not had previous methotrexate treatment. Arthritis Rheum. 2006;54:26-37.

5. Bakker MF, Jacobs JW, Welsing PM, Verstappen SM, Tekstra J, Ton E et al. Low-dose prednisone inclusion in a methotrexate-based, tight control strategy for early rheumatoid arthritis: a randomized trial. Ann Intern Med. 2012;156:329-39.

6. Soubrier M, Puechal X, Sibilia J, Mariette X, Meyer O, Combe B, et al. Evaluation Of two strategies (initial methotrexate monotherapy vs its combination with adalimumab) in management of early active rheumatoid arthritis: data from the Guepard trial. Rheumatology (Oxford). 2009:48:1429-34.

7. Soubrier M, Puechal X, Sibilia J, Mariette X, Meyer O, Combe B et al. Efficacy and safety of ascending methotrexate dose in combination with adalimumab: the randomised Concerto trial. Ann Rheum Dis. 2015;74:1037-44.

8. Bergstra SA, Allaart CF, Stijnen T, Landewe RBM. Meta-regression of a doseresponse relationship of methotrexate in mono- and combination therapy in DMARD naive early rheumatoid arthritis patients. Arthritis Care Res (Hoboken). 2017;69:1473-83.

9. van den Berg R, Van Der Heijde D, Landewe RBM, van Lambalgen K, Huizinga TWJ. The Meteor initiative: the way forward for optimal, worldwide data integration to improve care for RA patients. Clin Exp Rheumatol. 2014; 32:S-135-40.

10. Brookhart MA, Schneeweiss S, Rothman KJ, Glynn RJ, Avorn J, Stürner T. Variable selection for propensity score models. Am J Epidemiol. 2006;163: 1149-56.

11. Mottonen T, Hannonen $\mathrm{P}$, Leirisalo-Repo M, Nissila M, Kautiainen H, Korpela $M$ et al. Comparison of combination therapy with single-drug therapy in 
early rheumatoid arthritis: a randomised trial. Fin-Raco Trial Group Lancet. 1999:353:1568-73.

12. Dervieux T, Zablocki R, Kremer J. Red blood cell methotrexate polyglutamates emerge as a function of dosage intensity and route of administration during pulse methotrexate therapy in rheumatoid arthritis. Rheumatology (Oxford, England). 2010;49:2337-45.

13. Verschueren P, De Cock D, Corluy L, Joos R, Langenaken C, Taelman V et al. Methotrexate in combination with other DMARDS is not superior to methotrexate alone for remission induction with moderate-to-high-dose glucocorticoid bridging in early rheumatoid arthritis after 16 weeks of treatment: The Carera Trial. Ann Geophys. 2015;74:27-34.

14. Fleischmann R, Weinblatt M, Schiff M, Khanna D, Maldonado M, Nadkarni A, et al. Patient-reported outcomes from a two-year head-to-head comparison of subcutaneous abatacept and adalimumab for rheumatoid arthritis. Arthritis Care Res (Hoboken). 2016:68:907-13.

15. Van der Kooij SM, de Vries-Bouwstra JK, Goekoop-Ruiterman YP, van Zeben D, Kerstens PJ, Gerards AH et al. Limited efficacy of conventional DMARDS after initial methotrexate failure in patients with recent onset rheumatoid arthritis treated according to the disease activity score. Ann Rheum Dis. 2007;66:1356-62.

Submit your next manuscript to BioMed Central and we will help you at every step:

- We accept pre-submission inquiries

- Our selector tool helps you to find the most relevant journal

- We provide round the clock customer support

- Convenient online submission

- Thorough peer review

- Inclusion in PubMed and all major indexing services

- Maximum visibility for your research

Submit your manuscript at www.biomedcentral.com/submit
Biomed Central 